Draft Version June 28, 2018

Preprint typeset using IATEX style emulateapj v. 03/07/07

\title{
EARLY-TYPE HOST GALAXIES OF TYPE II AND IB SUPERNOVAE
}

\author{
Hyewon Suh ${ }^{1}$, Sung-Chul Yoon ${ }^{2}$, Hyunjin JeOng ${ }^{1}$, Sukyoung K. Yi ${ }^{1}$ \\ Draft version June 28, 2018
}

\begin{abstract}
Recent studies find that some early-type galaxies host Type II or Ibc supernovae (SNe II, Ibc). This may imply recent star-formation activities in these SNe host galaxies, but a massive star origin of the SNe Ib so far observed in early-type galaxies has been questioned because of their intrinsic faintness and unusually strong $\mathrm{Ca}$ lines shown in the nebular phase. To address the issue, we investigate the properties of early-type SNe host galaxies using the data with Galaxy Evolution Explore (GALEX) ultraviolet photometry, and the Sloan Digital Sky Survey (SDSS) optical data. Our sample includes eight SNe II and one peculiar SN Ib (SN 2000ds) host galaxies as well as 32 SN Ia host galaxies. The host galaxy of SN 2005cz, another peculiar SN Ib, is also analysed using the GALEX data and the NASA/IPAC Extragalactic Database (NED) optical data. We find that the NUV-optical colors of SN II/Ib host galaxies are systematically bluer than those of SN Ia host galaxies, and some SN II/Ib host galaxies with NUV-r colors markedly bluer than the others exhibit strong radio emission. We perform a stellar population synthesis analysis and find a clear signature of recent star-formation activities in most of the SN II/Ib host galaxies. Our results generally support the association of the SNe II/Ib hosted in early-type galaxies with core-collapse of massive stars. We briefly discuss implications for the progenitors of the peculiar SNe Ib 2000ds and $2005 \mathrm{cz}$.
\end{abstract}

Subject headings: galaxies: elliptical and lenticular, cD - galaxies: evolution - supernovae: general

\section{INTRODUCTION}

There is a general consensus that most (if not all) $\mathrm{SNe}$ II, Ib and Ic originate from the core-collapse of massive stars. Observations show that they occur predominantly in late-type galaxies where active starformation takes place (e.g., Maza \& van den Bergh 1976; Cappellaro et al. 1999; Hamuy 2003). However, several studies report observations of SNe II and Ibc in earlytype galaxies (van den Bergh et al. 2002, 2003, 2005; Hakobyan et al. 2008; Leaman et al. 2010), which are traditionally considered old stellar populations composed mainly of low-mass stars. Although Hakobyan et al. (2008) argue that many of the previously reported earlytype SN II/Ib host galaxies may be misclassified spirals, it is clear that at least some of them belong to genuine early-types in the morphological sense (Hakobyan et al. 2008; Leaman et al. 2010).

Perets et al. (2010) note that all of the SNe Ibc observed so far in early-type galaxies belong to a subclass of Type Ib supernovae; they show a typical Type Ib like spectrum characterized by the strong helium lines and weak silicon lines at early time, but they appear to be systematically fainter than typical $\mathrm{SNe} \mathrm{Ib}$, and show strong $\mathrm{Ca}$ II and weak O I emission lines in late-time optical spectra. Their origin is currently a matter of debate. Their progenitors might be related to rather old stellar populations (e.g., helium-accreting white dwarfs or mergers of $\mathrm{ONeMg}-$ and He white dwarfs; Kawabata et al. 2010; Perets et al. 2010), or their relatively frequent detection in early-type galaxies might be due to their intrinsic faintness, even if they have a core-collapse origin

\footnotetext{
${ }^{1}$ Department of Astronomy, Yonsei University, Seoul 120-749, Korea; yi@yonsei.ac.kr

2 Argelander Institute for Astronomy, University of Bonn, Auf dem Huegel 71, D-53121 Bonn, Germany
}

(Kawabata et al. 2010).

Given this intriguing finding, a careful study of the stellar population in the early-type host galaxies of $\mathrm{SNe}$ $\mathrm{II} / \mathrm{Ibc}$ is important for a better understanding of recent star formation activities that may be related to such events. The Galaxy Evolution Explorer (GALEX) ultraviolet (UV) filters are particularly useful for this purpose, since they allow us to detect even a tiny amount of young massive stars in galaxies. Many recent studies with GALEX data show that a significant fraction of early-type galaxies exhibit enhanced UV light as a sign of recent star formation (Yi et al. 2005; Salim et al. 2007; Donas et al. 2007; Schawinski et al. 2007a; Kaviraj et al. 2007, 2008).

In this paper, we present the UV-optical colormagnitude relation of early-type host galaxies of some SNe II/Ib, including SN 2000ds and SN 2005cz, which belong to the faint, Ca-rich class. By comparing them to early-type host galaxies of SNe Ia, we discuss whether the properties of early-type SN II/Ib host galaxies differ systematically from those of SNe Ia, and if the faint, Ca-rich class of SN Ib can still be explained within the framework of the core-collapse scenario.

Throughout this paper we assume a $\Lambda \mathrm{CDM}$ cosmology with $\Omega_{m}=0.3$ and $\mathrm{H}_{0}=70 \mathrm{~km} \mathrm{~s}^{-1} \mathrm{Mpc}^{-1}$.

\section{SAMPLE}

The catalogue of the Center for Astrophysics (CfA) provides all supernovae ${ }^{3}$ reported since 1885 . We construct a list of galaxies that have hosted SNe II or Ibc in this catalogue that overlap with Data Release 7 of the Sloan Digital Sky Survey (SDSS, York et al. 2000;

\footnotetext{
${ }^{3}$ http://www.cfa.harvard.edu/iau/lists/Supernovae.html
} 
TABLE 1

SAMPLE

\begin{tabular}{|c|c|c|c|c|c|c|c|c|c|c|}
\hline \multicolumn{3}{|c|}{ Host galaxy } & \multicolumn{8}{|c|}{ Supernova } \\
\hline 2 & SDSS J160713.55-000443.6 & 0.031 & 5.61 & 5.51 & - & $2001 \mathrm{ax}$ & SNII & 17.5 & 2.57 & \\
\hline 3 & NGC 774 & 0.015 & 13.78 & 5.24 & - & 2006 ee & SNII & 17.6 & 3.07 & \\
\hline 4 & NGC 2768 & 0.005 & 29.67 & 4.63 & 20.75 & $2000 \mathrm{ds}$ & SNIb & 17.9 & 2.23 & "Ca-rich" \\
\hline 5 & SDSS J024606.79-073803.7 & 0.030 & 6.00 & 4.28 & - & 2008al & SNII & 17.6 & 12.89 & \\
\hline 8 & SDSS J164734.90+495000.7 & 0.048 & 1.85 & 3.38 & 23.90 & $2009 \mathrm{fe}$ & SNII & 18.1 & 0.65 & \\
\hline 9 & SDSS J003328.04-001912.9 & 0.107 & 2.79 & 2.67 & 22.41 & 2006ho & SNII & 19.6 & 0.05 & \\
\hline
\end{tabular}

a Redshift

b Apparent magnitude of the supernova

c Distance between the center of the host galaxy and the supernova position

Stoughton et al. 2002; Abazajian et al. 2009). We crossmatch the detections in the GALEX GR5/6 archive, and perform visual inspection of both SDSS optical and GALEX UV images to select early-type galaxies. We de-redden the colors with respect to Galactic extinction using Schlegel et al. (1998) maps provided by the SDSS pipeline and assuming $\mathrm{A}_{N U V}=8.741 \times \mathrm{E}(\mathrm{B}-\mathrm{V})$ (Wyder et al. 2005). Thus, the final sample comprises 9 early-type host galaxies of $\mathrm{SNe} \mathrm{II} / \mathrm{Ib}^{4}$. We also use radio observations in the Faint Images of the Radio Sky at Twenty-cm (FIRST) survey (Becker et al. 1995), which has an angular resolution of $\sim 5$ " and a completeness limit of $1 \mathrm{mJy}$. There are 3 sample galaxies with radio detections. For comparison, we use the same procedure to construct a sample of early-type galaxies hosting a SN Ia.

We list the SNe II/Ib sample in Table1, which includes one confirmed SN Ib and eight SNe II. All SNe II in our sample (except for SN 2006gy) seem to have luminosity typical of core collapse supernovae (see Table 1); thus, they are not likely to belong to a hybrid class of SN Ia. Figure 1 shows a sample of early-type host galaxies of $\mathrm{SNe} \mathrm{II} / \mathrm{Ib}$ with optical, UV, and radio images. The SDSS optical images are shown in the top row, where the positions of SNe are marked by star symbols. The $G A L E X$ UV images and the FIRST radio images are shown in the second and third rows, respectively. Most of the $\mathrm{SN} \mathrm{II/Ib} \mathrm{reside} \mathrm{close} \mathrm{to} \mathrm{the} \mathrm{center} \mathrm{of} \mathrm{the} \mathrm{host}$ galaxy, except for SN 2008al. Their locations are similar to those indicated by $\mathrm{UV}$ and radio emission.

Regarding morphology of host galaxies, NGC 1260 (label 1) and NGC 774 (label 3) are classified as S0 in the NASA/IPAC Extragalactic Database (NED). NGC 2768 (label 4) is classified as elliptical. Labels 5, 7, 8, and 9 have fracDev $=1$, which is the weight of de Vaucouleurs profile in the best composite (de Vaucouleurs + exponential) fit to the image in $r$ band. Labels 2 and 6 have fracDev=0.89. All nine galaxies have concentration indices greater than 2.5. All galaxies are fairly bright, and all but one are close $(z<0.05)$; thus, morphology deter-

4 We exclude the SN 1986M, which is classified as SN $\mathrm{Ib}$ in the CfA catalogue and $\mathrm{SN}$ Ia in the Asiago SN catalogue (http://web.oapd.inaf.it/supern/cat/) respectively. Given its rather high luminosity, it is more likely to be a SN Ia rather than a SN Ib. mination based on visual inspection and pipeline parameters is deemed reliable.

\section{UV-OPTICAL COLOR-MAGNITUDE RELATION}

The UV-optical color magnitude relation (see, e.g., Yi et al. 2005; Kaviraj et al. 2007; Schawinski et al. 2007a) is a particularly efficient tool for studying recent star formation in early-type galaxies because of its high sensitivity to young stellar populations. Figure 2 shows the NUV $-\mathrm{r}$ color magnitude relation for the SN II host galaxies (blue circles with label), the SN Ib (2000ds) host galaxy (star symbol), and the SN Ia host galaxies (small red circles). We also show the host galaxy of SN $2005 \mathrm{cz}$ (NGC 4589; cf. Kawabata et al. 2010), with a star symbol. We do not have SDSS photometry of this galaxy, so we convert its Johnson magnitudes (from NED) to SDSS magnitudes using the equations given by Jester et al. (2005). We also show typical early-type galaxies (grey dots) for comparison. The empirical threshold of NUV $-\mathrm{r}$ $<5.4$ (dashed line) is a criterion that is used to find old galaxies with recent star formation. Yi et al. (2005) derived this value from the nearby prominent UV-upturn galaxy NGC 4552; hence, it should be considered a lower bound in the NUV $-\mathrm{r}$ color of purely-old stellar populations.

A cursory inspection of this diagram shows that most of the early-type host galaxies of SNe II/Ib reside below $\mathrm{NUV}-\mathrm{r} \sim 5.4$, implying that they have undergone recent star formation, while the SN Ia host galaxies show relatively redder UV-optical colors. We also find that $\mathrm{SNe} \mathrm{II} / \mathrm{Ib}$ preferentially occur in relatively small host galaxies $\left(M_{\mathrm{r}} \gtrsim-22\right)$ compared to the case of SNe Ia (cf. Arcavi et al. 2010). This is not surprising considering that supernova rates correlate with star formation rates. It should be noted that less massive early-type galaxies are more likely to have experienced recent star formation (Schawinski et al. 2007a; Jeong et al. 2009). Li et al. (2010) finds that SNe II preferentially occur in relatively smaller host galaxies. But direct comparison is difficult because interpretation on galaxy size can be tricky when dealing with a heterogeneous sample of galaxy morphologies.

The host galaxy of SN 2000ds (NGC 2768, label 4) shows a NUV $-\mathrm{r}$ color that is significantly bluer than those of most SN Ia hosts, strongly implying the pres- 

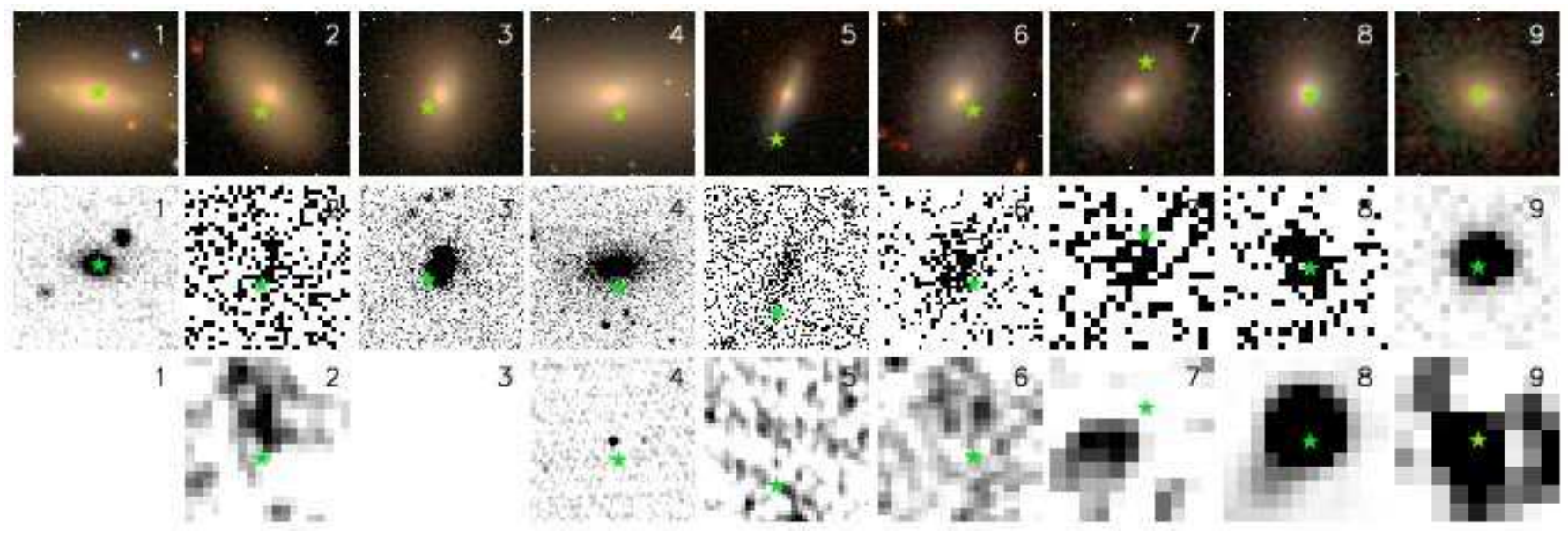

FIG. 1.- A sample of early-type host galaxies of SN II/Ib. The top row is SDSS optical images, the middle row is $G A L E X$ ultraviolet images, and the bottom row is FIRST radio images. The top optical images show the positions of supernovae marked by star symbols. We label the host galaxy listed in Table 1 in each panel.

ence of young stars. On the other hand, NGC 4589 (star symbol), which is the host galaxy of another peculiar SN Ib (SN 2005cz), shows a marginal NUV-r color. Note that NGC 4589 is a strong $21 \mathrm{~cm} \mathrm{HI}$ source (Theureau et al. 2007) and shows large opaque dust patches (Michard \& Marchal 1994; Carollo et al. 1997; Lauer et al. 2005). Also note that NUV colors are more sensitive to dust than optical colors by a factor of 4 , so the presence of a small amount of dust can have a proportionally greater effect in the NUV than in the optical colors. After all, the intrinsic colors of this galaxy might be substantially bluer than the observed colors.

Our sample includes the host galaxy of SN 2006gy (NGC 1260, label 1), which is one of the most luminous supernovae ever discovered. Even though the progenitor of this highly luminous SN IIn is generally believed to be a very massive star (e.g., Smith et al. 2010), the NUV $-r$ color of the host galaxy is not particularly blue compared to the other SN II/Ib host galaxies. Like NGC 4589 , this galaxy has also been found to be fairly dusty (Ofek et al. 2007).

We find that the host galaxy of SN 2000ds and two of the eight SN II host galaxies that show a markedly bluer NUV-r color (labels 8 and 9) are strong radio sources (see Table [1] and Figure 11). Recently, some authors argued that radio loudness in early-type galaxies may be related to a recent merger event that enhanced the SN Ia rate from young stellar populations (Della Valle et al. 2005; Mannucci et al. 2006; Graham et al. 2010). If this is the case, the strong radio emission in these galaxies would serve as more evidence of recent star formation in these host galaxies. However, not all SN II host galaxies in our sample have significant radio emission. The issue of the correlation between radio emission and the presence of young stellar populations should be addressed carefully in future work.

\section{STELLAR POPULATION ANALYSIS}

The use of NUV $-\mathrm{r}$ color to find galaxies with recent star formation (as discussed in the previous section) is overly simplistic, as it is based on the assumption that all early-type galaxies should have an underlying UVupturn component from old stars at a level similar to that of NGC 4552 (see the review of O'Connell 1999).
Contrarily, it was recently found that only a small fraction of early-type galaxies show a UV upturn (Yi 2010). Hence, we hereby use a more sophisticated method to characterize the stellar populations in our SN host galaxies.

We consider a two-stage star formation history to determine the age and mass fraction of the young stellar component in the SN host galaxies. Both starbursts are assumed to be instantaneous. The base models in this study are taken from Yi (2003), which are specialized for old stellar populations. Since these models do not cover ages younger than $1 \mathrm{Gyr}$, we combine them with the models of Bruzual \& Charlot (2003) for a young stellar population (Ferreras \& Silk 2000; Kaviraj et al.|2007; Schawinski et al. 2007b). We assume a single uniform age of 12 Gyr for the old component. The young component is allowed to vary in age $\left(10^{-3} \leq t_{\text {young }} \leq 10\right.$ Gyr $)$ and mass fraction $\left(10^{-6} \leq f_{\text {young }} \leq 1\right)$. More complex models, such as those based on multiple star bursts or continuous star formation, do not affect this analysis very much because UV lights are insensitive to the details of the star formation history, but are highly dominated by the most recent star formation event.

To constrain these two parameters, we fit the observed UV and optical colors to models, and compute the associated $\chi^{2}$ statistic to obtain a probability distribution of the age and mass fraction of the young stellar component. Internal extinction is also constrained in the $\chi^{2}$ minimization. The best fit and confidence levels are shown in Figure 3. The $\chi^{2}$ minimum is marked with circles along with error bars.

We first note that this analysis is heavily subject to various degeneracies. The most important one for the UV analysis is the age-mass degeneracy. A larger fraction of the young component is hardly distinguishable from a smaller fraction of the younger component.

The majority of SN Ia host galaxies have a characteristic age of $3-610^{8}$ yr for the young stellar component, which agrees with previous studies (e.g. Mannucci et al. 2005; Sullivan et al. 2006; Schawinski 2009). Our quiescent SN Ia host galaxies (filled red circles) are found to have a small amount of young stars, but this is because our building-block population models do not have a significant UV flux such as that exhibited by UV up- 


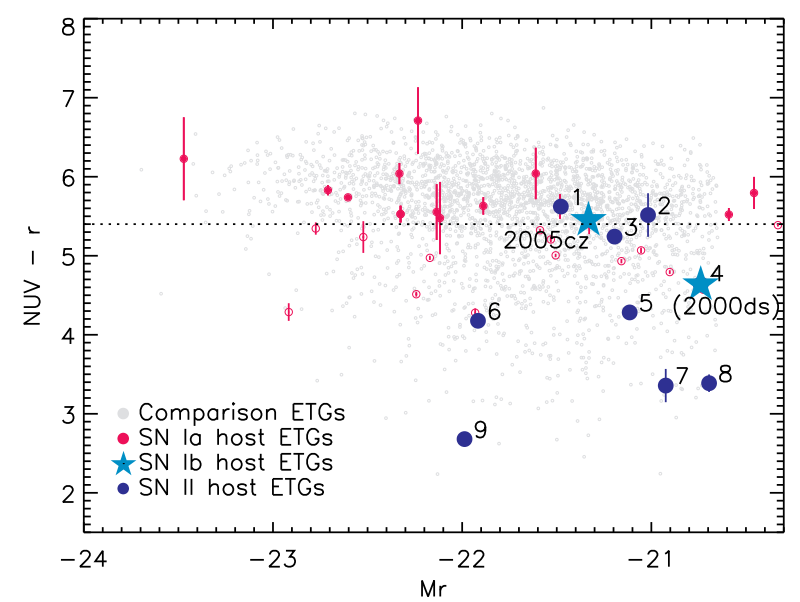

FIG. 2.- The UV-optical color-magnitude relation. We plot early-type host galaxies of SNe II and SNe Ia with blue and red circles, respectively. The dotted line shows the NUV $-\mathrm{r}=5.4$ cut, which indicates the color of the nearby UV-upturn galaxy, NGC 4552 (see the text for details). We mark the SN Ia hosts with a color above/below this cut by filled/open red circles. Star symbols denote SN Ib host galaxies. The grey points are the comparison sample of early-type galaxies from SDSS-GALEX crossmatches (that is, without a detected SN). We also label each host galaxy listed in Table 1

turn galaxies (e.g., NGC 4552). These galaxies usually have a small UV flux, with which robust estimations of the young component properties are difficult. They lie along the degeneracy area marked by the pink-shaded region. In this regard, the young component mass fraction is an upper limit in the case of SN Ia host galaxies. Note that the SN Ia host galaxies with a blue UV-optical color (open red circles) are mostly below this shade, indicating either a greater amount of young stars or a younger age, both of which produce a higher UV flux.

The SN II/Ib host galaxies are markedly different from the quiescent SN Ia host galaxies. Most of the SN $\mathrm{II} / \mathrm{Ib}$ host galaxies are located below the degeneracy region, strongly indicating the presence of young stars.

\section{IMPLICATIONS FOR THE PROGENITORS} OF FAINT, CA-RICH SNE IB

The SNe II in our sample must have a core-collapse origin as mentioned in $\S 2$, and the above discussion confirms that their progenitor ages are systematically smaller than those of the SNe Ia. The host galaxy of SN 2000ds (NGC 2768) appears to have properties similar to those of the SNe II host galaxies. The relatively strong radio emission of NGC 2768 may be additional evidence to support the connection between SN 2000ds and recent star formation. The host galaxy of SN $2005 \mathrm{cz}$ (NGC4589) also seems to show signatures of recent star formation. It is located below the degeneracy region in Figure 3 and known to be dusty as discussed above. Our result thus indicates that the faint, Ca-rich SNe Ib 2000ds and $2005 \mathrm{cz}$ originate from massive stars like the SNe II in our sample.

Our result may give some hints for their progenitor masses. Given the characteristic age of about $300-400$ Myr for the young stellar component in NGC 2768 and NGC 4589 according to our stellar population model, the progenitors of SN 2000ds and SN $2005 \mathrm{cz}$ are not likely

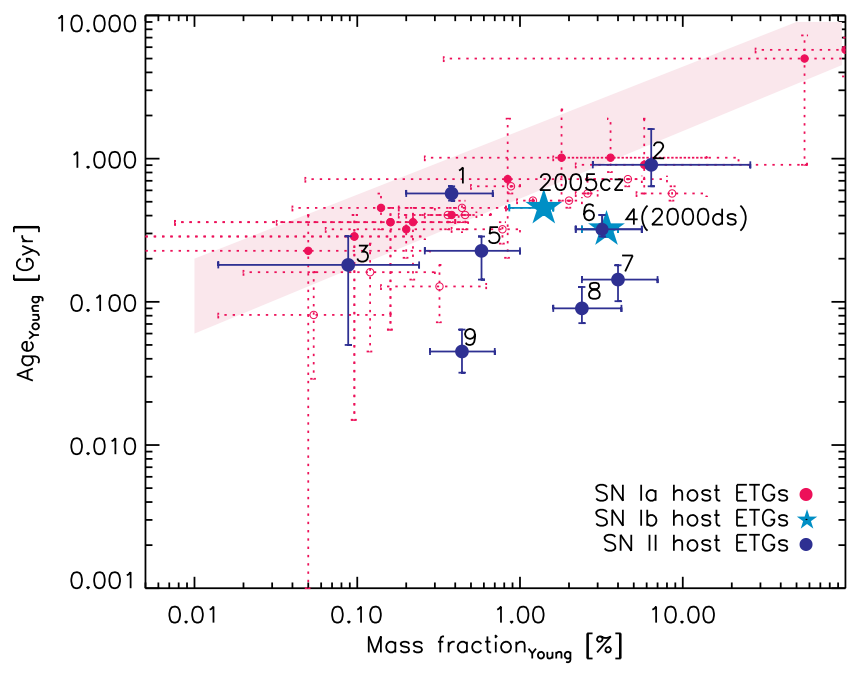

FIG. 3.- The distribution in age and mass fraction for the young stellar component. Blue and red symbols represent the best fit of early-type host galaxies of SNe II and SNe Ia, respectively. Star symbols indicate the SN Ib host galaxies. The pink-shaded region marks the age-mass degeneracy area. See the text for details.

to have an initial mass much larger than about $10 M_{\odot}$ This conclusion is very similar to that of Kawabata et al. (2010) for SN 2005cz. They argued that the high Ca to Oxygen abundance ratio implied by observation in the ejecta of such peculiar SNe Ib can be best explained by explosive nucleosynthesis in a relatively small stellar core containing very thin silicon and oxygen shells. The corresponding helium core size may not significantly exceed $3 M_{\odot}$. This independently points to moderately massive progenitors having initial masses of about $10 M_{\odot}$.

Both being SNe Ib, the progenitors of SN 2000ds and SN 2005cz must have lost their hydrogen envelope, leaving naked helium cores of $2-3 M_{\odot}$. This could be only possible in close binary systems, unless the mass loss rate of their progenitors were unrealistically high (e.g., Kawabata et al. 2010; Yoon et al. 2010). It is important to note that the initial masses of core collapse supernovae could be lower down to about $5-6 M_{\odot}$ with binary interactions, compared to the canonical limit of $8-10 M_{\odot}$ for single stars. Such binary star evolution with initial masses of $5-11 M_{\odot}$ towards helium stars of about $2-3 M_{\odot}$ such as SN Ibc progenitors is required to explain the formation of binary pulsars PSR B2303+46 and PSR J1141-6545 with old white dwarf companions (e.g. Tauris \& Sennels 2000). Therefore, it is not surprising that SNe Ib like 2000ds can be found in some early-type galaxies, hovering around relatively young stars with ages of $\sim 10^{8} \mathrm{Myr}$.

\section{ACKNOWLEDGMENTS}

This project made use of the SDSS optical data, the $G A L E X$ ultraviolet data, the HyperLeda database and the NASA/IPAC Extragalactic Database. This work was supported by the National Research Foundation of Korea through the Doyak grant (No. 20090078756) and the SRC grant to the Center for Galaxy Evolution Research. 


\section{REFERENCES}

Abazajian, K. N., et al. 2009, ApJS, 182, 543

Arcavi et al. 2010, ApJ, 721, 777

Becker, R. H., White, R. L., \& Helfand, D. J. 1995, ApJ, 450, 559

Bruzual, G., \& Charlot, S. 2003, MNRAS, 344, 1000

Cappellaro, E., Evans, R., \& Turatto, M. 1999, A\&A, 351, 459

Carollo, C. M., Franx, M., Illingworth, G. D., \& Forbes, D. A. 1997, ApJ, 481, 710

Della Valle, M., \& Panagia, N. 2003, ApJ, 587, L71

Della Valle, M., et al. 2005, ApJ, 629, 750

Donas, J., et al. 2007, ApJS, 173, 597

Ferreras, I., \& Silk, J. 2000, ApJ, 541, L37

Graham et al., 2010, ApJ, 139, 594

Hakobyan, A. A., Petrosian, A. R., McLean, B., Kunth, D., Allen,

R. J., Turatto, M., Barbon, R. 2008, A\&A, 488, 523

Hamuy, M. 2003, ApJ, 582, 905

Jester, S., et al. 2005, AJ, 130, 873

Jeong, H., et al. 2009, MNRAS, 398, 2028

Kaviraj, S., et al. 2007, ApJ, 173, 642

Kaviraj, S., et al. 2008, MNRAS, 388, 67

Kawabata, K. S., et al. 2010, nature, 465, 326

Leaman, J., Li, W., Chornock, R., Filippenko, A. V. 2010, arXiv:1006.4611

Lauer, T. R., Faber, S. M., Gebhardt, K., et al. 2005, AJ, 129, 2138

Li, W., et al. 2010, arXiv: 1006.4612v2

Mannucci, F., Della Valle, M., Panagia, N., Cappellaro, E., Cresci, G., Maiolino, R., Petrosian, A., \& Turatto, M. 2005, A\&A, 433, 807

Mannucci, F., Della Valle, M., \& Panagia, N. 2006, MNRAS, 370, 773

Maza, J., \& van den Bergh, S. 1976, ApJ, 204, 519

Michard, R., \& Marchal, J. 1994, A\&AS, 105, 481
O'Connell, R. W. 1999, ARA\&A, 37, 6030

Ofek, E. O., et al. 2007, ApJ, 659, L13

Perets, H. B., et al. 2010, nature, 465, 322

Salim, S., et al. 2007, ApJS, 173, 267

Schawinski, K. 2009, MNRAS, 397, 717

Schawinski, K., et al., 2007a, ApJS, 173, 512

Schawinski, K., et al., 2007b, MNRAS, 382, 1415

Schlegel, D. J., Finkbeiner, D. P, \& Davis, M. 1998, ApJ, 500, 525

Smith, N., Chornock, R., Silverman, J.M., Filippenko, A.V., \& Foley, R.J., 2010, ApJ, 709, 856

Stoughton, C., et al. 2002, AJ, 123, 485

Sullivan, M., et al. 2006, ApJ, 648, 868

Tauris, T.M., \& Sennels, T., 2000, A\&A, 355, 236

Theureau, G., Hanski, M. O., Coudreau, N., Hallet, N., \& Martin, J.-M. 2007, A\&A, 465, 71

van den Bergh, S., Li, W., \& Filippenko, A. V. 2002, PASP, 114, 820

van den Bergh, S., Li, W., \& Filippenko, A. V. 2003, PASP, 115, 1280

van den Bergh, S., Li, W., \& Filippenko, A. V. 2005, PASP, 117, 773

Wyder, T. K., et al., 2005, ApJ, 619, L15

Yi, S. K. 2003, ApJ, 582, 202

Yi, S. K., et al. 2005, ApJ, 619, L111

Yi, S. K. 2010, in Stellar Populations: Planning for the Next Decade IAU Symposium No. 262, (Cambridge University Press: Cambridge), 147

Yoon, S.-C., Woosley, S.E., \& Langer, N. 2010, ApJ, 725, 940

York, D. G., et al. 2000, AJ, 120, 1579 